\title{
Electronic versus phononic friction of xenon on silver
}

\author{
A. Liebsch* \\ Institut für Festkörperforschung, Forschungszentrum Jülich, 52425 Jülich, Germany \\ S. Gonçalves ${ }^{\dagger}$ \\ Instituto de Física, Universidade Federal do Rio Grande do Sul, Caixa Postal 15051, 91501-970 Porto Alegre (RS), Brazil \\ M. Kiwi \\ Facultad de Física, Pontificia Universidad Católica, Casilla 306, Santiago 22, Chile
}

(Received 28 July 1998; revised manuscript received 11 March 1999)

\begin{abstract}
Molecular dynamics simulations of a Xe monolayer sliding on $\mathrm{Ag}(001)$ and $\mathrm{Ag}(111)$ are carried out in order to ascertain the microscopic origin of friction. For several values of the electronic contribution to the friction of individual $\mathrm{Xe}$ atoms, the intraoverlayer phonon dissipation is calculated as a function of the corrugation amplitude of the substrate potential, which is a pertinent parameter to consider. Within the accuracy of the numerical results and the uncertainty with which the values of the relevant parameters are known at present, we conclude that electronic and phononic dissipation channels are of similar importance. While phonon friction gives rise to the rapid variation with coverage, the electronic friction provides a roughly coverage-independent contribution to the overall sliding friction. [S0163-1829(99)14231-0]
\end{abstract}

\section{INTRODUCTION}

The microscopic origin of the sliding friction of thin films adsorbed on a metal surface has recently attracted considerable interest. ${ }^{1,2}$ The availability of refined experimental methods ${ }^{3-5}$ makes it now feasible to investigate the fundamental processes that contribute to the sliding friction on an atomic scale. On a metal surface, frictional energy is dissipated via two main channels: excitation of low-energy electron-hole pairs in the substrate ${ }^{6-12}$ and excitation of phonons within the overlayer. ${ }^{13-16}$ Phonon excitation within the metal may also occur; however, this contribution usually has only a minor influence on the lateral sliding of adsorbed films.

Detailed experimental information on the friction of Xe and $\mathrm{Kr}$ layers on noble metal surfaces was recently obtained by Krim et $a l .^{3,4}$ who performed quartz-crystal microbalance (QCM) measurements for a variety of coverages. In this technique, the frequency shift and broadening of the resonant line provide information on the number of adsorbed atoms and on their slippage during the lateral oscillation of the microbalance. The data were analyzed by various theoretical groups that, surprisingly, arrived at contradictory conclusions: For $\mathrm{Kr}$ on $\mathrm{Au}(111)$, Cieplak et al. ${ }^{13,14}$ argued that the observed friction is caused by lattice vibrations within the overlayer and that substrate-induced energy dissipation plays a negligible role. Dominant phonon damping was also inferred for Xe on $\operatorname{Ag}(111)$ by Tomassone et al. ${ }^{16}$ On the other hand, Persson et al. ${ }^{15}$ argued that the phonon-associated friction for a full Xe monolayer on $\operatorname{Ag}(100)$ is small and that the measured friction is mainly of electronic origin. These opposite interpretations are puzzling since all three theoretical studies were based on molecular dynamics simulations for similar models of the rare-gas-metal-adsorption system. In addition, Liebsch ${ }^{11,12}$ performed dynamical surface response calculations within the time-dependent density functional ap- proach to determine the electronic friction of $\mathrm{Xe}$ atoms on Ag. The lateral component of the friction coefficient was shown to agree qualitatively with the experimental value for a full monolayer, suggesting that phonon processes play a minor role in the compressed phase.

In view of these fundamental theoretical discrepancies, we decided to carry out molecular dynamics simulations for $\mathrm{Xe}$ on $\mathrm{Ag}$, employing essentially the same overall model as previous authors. ${ }^{13-16}$ Key input parameters in these simulations are, in particular, the corrugation amplitude of the $\mathrm{Xe} / \mathrm{Ag}$ interaction potential and the electronic friction of individual Xe atoms sliding parallel to the substrate surface. Since at present the corrugation amplitude is known from independent experimental data only with very appreciable uncertainty, a detailed understanding of how its magnitude influences the final result is in our view crucial. In contrast to the earlier molecular dynamics studies, therefore, we do not choose a specific set of input model parameters but investigate the net sliding friction within a wide range of these parameters. In addition, we consider the dependence on the crystallographic orientation by studying the friction of a Xe layer adsorbed on $\operatorname{Ag}(111)$ and $\operatorname{Ag}(100)$, in order to check the importance of the lateral symmetry of the substrate potential. By carrying out such a systematic investigation we essentially cover the full range of overlayer-substrate models considered in the previous treatments.

A striking feature of the Xe/Ag quartz-crystal microbalance data by Krim et $a .^{3,4}$ is the strong variation of the net sliding friction with Xe coverage. To facilitate the discussion, we show in Fig. 1 the measured slip time $\tau$ as a function of Xe coverage ${ }^{4}$ on $\operatorname{Ag}(111)$. The friction coefficient $\bar{\eta}$ is given by the inverse of the slip time. According to surface resistivity measurements for $\mathrm{Xe}$ on $\mathrm{Ag},{ }^{5}$ the electronic friction coefficient depends weakly on coverage. It is evident, therefore, that the rapid coverage dependence observed in the QCM data, in particular near completion of a monolayer, is 


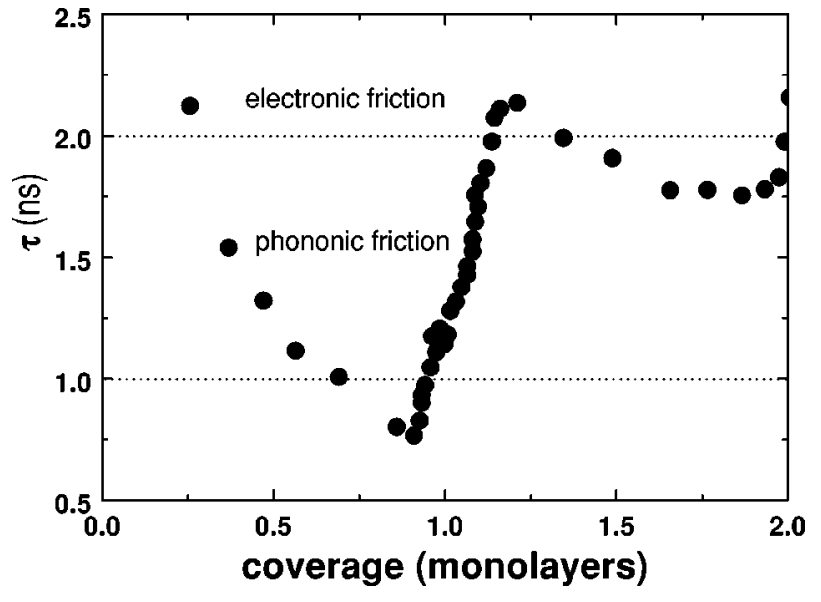

FIG. 1. Measured slip time $\tau$ for $\mathrm{Xe}$ on $\operatorname{Ag}(111)$, as a function of coverage, after Ref. 4. In this paper we put forward arguments, based on extensive simulations, to show that the region roughly between the dotted lines can be understood as due to phonon originated friction, while above the upper dotted line a friction mechanism of electronic origin seems to prevail.

caused primarily by phonons generated within the overlayer. This is not surprising since the probability of exciting phonons varies greatly with interadatom spacing. With respect to this aspect of the data, there is little dispute among the previous simulations studies.

The important question, rather, concerns the relative weights of electronic and phononic dissipation channels, especially at coverages near a full monolayer where the slip time reaches its maximum, i.e., where the sliding friction of the incommensurate overlayer is smallest. Our results show that the effective friction parameter $\bar{\eta}$ may be approximately written as

$$
\bar{\eta}=\eta_{\mathrm{el}}+\eta_{\mathrm{ph}} .
$$

Here, $\eta_{\mathrm{el}}=\eta_{\|}$is the parallel component of the single-atom electronic friction coefficient and the intra-adsorbate phonon contribution is roughly given by $\eta_{\mathrm{ph}}=c u_{0}^{2}$, where $u_{0}$ is the corrugation amplitude of the Xe/Ag potential. A quadratic variation with $u_{0}$ agrees with the analytical and numerical results of Cieplak et al. ${ }^{13,14}$ The coefficient $c$ depends weakly on the crystal face and on the single-atom friction parameters $\eta_{\|}$and $\eta_{\perp}$, but varies strongly with coverage. Evidently, according to the above expression, the measured sliding friction at any given coverage can be reproduced using several combinations of the parameters $u_{0}$ and $\eta_{\mathrm{el}}$. For instance, by reducing the single-site electronic friction $\eta_{\|}$, one is able to match the data if the substrate potential is assumed to be sufficiently strongly corrugated. Conversely, if the electronic friction is chosen large enough, little additional phonon damping is required to reproduce the same net $\bar{\eta}$. Constraints on the range of acceptable parameter values are implied by the observed variation of the slip time with coverage, in particular, by the steep slope near completion of the first monolayer.

The molecular dynamics simulations for Xe on Ag, which we describe below, suggest that the characteristic coverage dependence of the observed slip time and its overall magnitude cannot be understood only in terms of phonon dissipa- tion. Although there is considerable uncertainty both in the calculated and measured friction coefficients, the most likely scenario is the presence of a roughly coverage-independent electronic contribution of about $\tau \approx 2 \mathrm{~ns}\left(\eta_{\|} \approx 0.5 \mathrm{~ns}^{-1}\right)$ and an additional, strongly coverage-dependent phonon friction, which can reduce the slip time to about $\tau \approx 1 \mathrm{~ns}$ (the net sliding friction then increases approximately to $\bar{\eta} \approx 1 \mathrm{~ns}^{-1}$ ). Thus, roughly speaking, we associate the slip time in the region between the horizontal dotted lines in Fig. 1 with phonon processes, while the portion above the upper line is related to electronic energy dissipation. Remarkably, the electronic friction deduced from this analysis is in qualitative agreement with independent surface resistivity data for Xe on Ag (Ref. 5) and with theoretical estimates of this dissipation channel. ${ }^{11,12}$ Thus, the friction of a full monolayer is essentially of electronic origin, while the rapid increase of the slip time before reaching the complete monolayer is due to excitation of phonons within the Xe overlayer.

This paper is organized as follows. Section II summarizes the theoretical results available for the electronic friction of $\mathrm{Xe}$ atoms on a metal surface. Section III presents the model employed in the molecular dynamics simulations. In particular, we specify the interaction potentials and the friction felt by a single Xe atom. Various technical aspects of the calculation of the phonon friction are also discussed. Section IV contains the results, with special emphasis on the variation of the net sliding friction with the corrugation of the substrate potential and its dependence on the electronic friction. Our conclusions are given in Sec. V.

\section{ELECTRONIC FRICTION}

We consider first the electronic contributions to the sliding friction of individual $\mathrm{Xe}$ atoms above a metal surface. In a physisorption system, these may arise due to the van der Waals attraction and the Pauli repulsion. In the case of Xe, some dissipation might also be associated with the formation of a weak covalent bond.

It is well known that the instantaneous mutual polarization between neutral species gives rise to the long-range van der Waals attraction. If an atom moves relative to a metal, the induced surface charge lags behind, causing kinetic energy to be dissipated. Thus, as pointed out long ago by various authors, ${ }^{6,7}$ the excitation of low-frequency electron-hole pairs in the surface region contributes to the friction of neutral atoms. If the adatom velocity is small compared to the Fermi velocity of the metal electrons, the friction coefficient may be expressed as ${ }^{7,9}$

$$
\eta_{i}=-\frac{e^{2}}{m} \lim _{\omega \rightarrow 0} \frac{1}{\omega} \int d^{3} r \int d^{3} r^{\prime} V_{i}(\mathbf{r}) \operatorname{Im} \chi\left(\mathbf{r}, \mathbf{r}^{\prime}, \omega\right) V_{i}\left(\mathbf{r}^{\prime}\right)
$$

The index $i=x, z$ refers to parallel or perpendicular motion of the atom, $\chi$ is the many-body response function of the metal, and $m$ is the adatom mass. The effective interaction $V_{i}(\mathbf{r})$ is approximately given by

$$
V_{i}(\mathbf{r})=\frac{\alpha(0)}{2} \nabla_{i}\left(\nabla \frac{1}{|\mathbf{r}-\mathbf{d}|}\right)^{2}
$$


Here, $\alpha(0)$ is the static polarizability of the adatom and $\mathbf{d}$ $=(0,0, d)$ is the location relative to the jellium edge. Since $V_{i}(\mathbf{r})$ does not satisfy the Laplace equation, it can only be approximately represented in terms of a superposition of evanescent plane waves of the form ${ }^{9}$

$$
V_{i}(\mathbf{r})=\sum_{\mathbf{q}_{\|}} V_{i}\left(\mathbf{q}_{\|}\right) e^{i \mathbf{q}_{\|} \cdot \mathbf{r}_{\|}+q z} .
$$

With this expansion we can write $\eta_{i}$ as

$$
\eta_{i}=\frac{e^{2}}{m} \frac{1}{2 \pi} \lim _{\omega \rightarrow 0} \frac{1}{\omega} \sum_{\mathbf{q}_{\|}}\left|V_{i}\left(\mathbf{q}_{\|}\right)\right|^{2} q \operatorname{Im} g(q, \omega),
$$

where the surface response function $g(q, \omega)$ is defined as

$$
g(q, \omega)=\int d z e^{q z} n_{1}(z, q, \omega)
$$

and $n_{1}(z, q, \omega)$ is the electronic surface density induced by an external potential given by $-(2 \pi / q) e^{i \vec{q}_{\|} \cdot \vec{r}_{\|}+q z}$.

In the case of $\mathrm{Xe}$ atoms physisorbed on $\mathrm{Ag}(111)$ at a temperature $T=77.4 \mathrm{~K}$, the distance $d=2.4 \AA$. The static polarizability of $\mathrm{Xe}$ is $\alpha(0)=4.0 \AA^{3}$. Using the density functional results for $n_{1}(z, q, \omega)$ and $g(q, \omega)$, we find $\eta_{\|}$ $\sim 0.34 \mathrm{~ns}^{-1}$ and $\eta_{\perp} \sim 1.74 \mathrm{~ns}^{-1} .11,12$

We note that the theoretical value of $\eta_{\|}$given above presumably represents a slight overestimate since the Xe atom is not completely outside the range of the electronic density profile as implicitly assumed in Eq. (2.4). Moreover, as a result of the hybridization between the $\mathrm{Ag} s$ and $d$ electrons, the surface polarizability of real Ag may be slightly smaller than that of the corresponding jellium model. ${ }^{17}$ We also point out that, according to Eq. (2.2), the friction coefficient varies asymptotically as $1 / d^{10}$, i.e., it is rather sensitive to the precise location of the adatom above the metal surface.

The friction coefficient arising from the van der Waals attraction derived above is significantly larger than the contribution due to the Pauli repulsion which was estimated by Persson $^{8}$ to be about $\eta_{\|} \sim 0.06 \mathrm{~ns}^{-1}$. In addition, for Xe there may exist some friction due to chemical effects resulting from the broadening of the $\mathrm{Xe} 6 s$ level. Estimates of this mechanism yield ${ }^{8} \eta \| 0.15 \mathrm{~ns}^{-1}$.

On the basis of these theoretical calculations and estimates, we conclude that the total electronic contribution to the lateral friction of single $\mathrm{Xe}$ atoms on $\mathrm{Ag}$ is approximately $0.5 \cdots 0.6 \mathrm{~ns}^{-1}$. At full monolayer coverage, this friction is presumably slightly smaller because of the weakening of the $\mathrm{Xe} / \mathrm{Ag}$ bonds. An electronic contribution of this magnitude is in excellent agreement with the surface resistivity measurements for Xe on Ag. ${ }^{5}$

According to our molecular dynamics simulations at $T$ $=77.4 \mathrm{~K}$, the distance of Xe atoms normal to the surface varies about $0.1 \AA$ around the equilibrium distance $d$ $=2.4 \AA$. For the asymptotic van der Waals attraction, such a variation implies a variation of $\eta_{\|}$roughly between 0.2 and $0.5 \mathrm{~ns}^{-1}$. For the Pauli and covalent-bond contributions the variation is exponential due to wave function overlap, with an exponent determined by the work function. This leads to a variation of $\eta_{\|}$of about $20 \%$. Thus, for some Xe atoms, the electronic friction is larger, for others smaller than the equilibrium value $0.5 \cdots 0.6 \mathrm{~ns}^{-1}$ quoted above. Because of this uncertainty the simulations are carried out for a sufficiently wide range of electronic friction coefficients.

\section{MODEL FOR PHONON FRICTION}

In this section we focus on the evaluation of the friction induced by the excitation of phonons within the adsorbed layer of Xe atoms. The single-atom frictional properties are assumed to be known and are used as inputs in the molecular dynamics simulation. We specify first the intra-adsorbate and adsorbate-substrate potentials and then discuss details concerning the molecular dynamics simulations.

\section{A. Potentials}

As in previous work, ${ }^{13-16}$ the interaction between Xe atoms is expressed as a sum of Lennard-Jones pair potentials

$$
\mathrm{V}(r)=\epsilon\left[\left(\frac{r_{0}}{r}\right)^{12}-2\left(\frac{r_{0}}{r}\right)^{6}\right],
$$

where $\epsilon=19 \mathrm{meV}$ is the well depth and $r_{0}=4.54 \AA$ is the interparticle spacing at the potential minimum. The total $\mathrm{Xe}-\mathrm{Xe}$ interaction potential then takes the form

$$
V=\frac{1}{2} \sum_{i \neq j} \mathrm{v}\left(\mathbf{r}_{i}-\mathbf{r}_{j}\right) .
$$

The adsorbate-substrate interaction potential is assumed to be given by

$$
U(\mathbf{r})=E_{0}\left[e^{-2 \alpha\left(z-z_{0}\right)}-2 e^{-\alpha\left(z-z_{0}\right)}\right]+u_{0} e^{-\alpha^{\prime}\left(z-z_{0}\right)} u(x, y),
$$

where for $\operatorname{Ag}(100)$

$$
u(x, y)=2+\cos (k x)+\cos (k y),
$$

whereas, in the case of $\operatorname{Ag}(111)$,

$$
\begin{aligned}
u(x, y)= & 1.5+\cos (2 k y / \sqrt{3})+\cos [k(x+y / \sqrt{3})] \\
& +\cos [k(x-y / \sqrt{3})] .
\end{aligned}
$$

Here, $k=2 \pi / a$ and $a=2.89 \AA$ is the spacing between neighboring $\mathrm{Ag}$ atoms. The unit vectors of the substrate lattice are $(a, 0),(0, a)$ for $\operatorname{Ag}(100)$ and $(a, 0), \quad(a / 2, a \sqrt{3} / 2)$ for $\mathrm{Ag}(111)$. The binding energy for $\mathrm{Xe}$ on $\mathrm{Ag}$ is $E_{0}=0.23 \mathrm{eV}$. The decay constant $\alpha$ may be deduced from the frequency of the perpendicular vibration, ${ }^{15}$ i.e., $\alpha=0.72 \AA^{-1}$. The decay constant $\alpha^{\prime}$ of the Fourier component should in principle be larger than $\alpha$. However, this difference is not very important and we choose $\alpha^{\prime}=\alpha$. The corrugation factor $u(x, y)$ is assumed to vanish at hollow adsorption sites. The potential barrier between neighboring hollow sites is $2 u_{0}$ for the (100) face and $0.5 u_{0}$ for the (111) face. The total barrier between hollow sites across the substrate lattice sites is $4 u_{0}$ for the (100) face and $4.5 u_{0}$ for (111).

The adsorbate-substrate interaction specified in Eq. (3.3) has the same form as the one used by Persson and Nitzan. ${ }^{15}$ Cieplak et al. ${ }^{13,14}$ and Tomassone et al..$^{16}$ on the other hand, used the expression derived by Steele ${ }^{18}$ for a sum of pair potentials. Since this potential is much more corrugated than the true interaction between rare gas atoms and metal surfaces, the coefficient of the nonvanishing Fourier component 
was drastically scaled down. The functional form of this potential also differs from expression (3.3). However, the precise shape near the potential minimum should not be crucial, as long as the overall corrugation is the same.

Since the parameter $u_{0}$ is decisive for the excitation of phonons within the overlayer, we do not choose a particular value. Instead, we calculate the net sliding friction as a function of $u_{0}$ in order to illustrate its sensitivity to the adsorbatesubstrate potential. In this manner, we cover the various substrate potentials considered previously.

\section{B. Simulations}

There are several methods of extracting the friction parameter from the molecular dynamics simulations. (i) One can apply a constant lateral force $\mathbf{F}$ to all the adatoms, while keeping the substrate atoms at rest. After an appropriate number of time steps, the steady-state velocity $\mathbf{v}$ of the overlayer is determined. If the friction is viscous, $\mathbf{v}$ satisfies the linear relation

$$
\mathbf{F}=m \bar{\eta} \mathbf{v}
$$

where $m$ is the adatom mass. This procedure was used by Cieplak et al. ${ }^{13,14}$ and by Persson and Nitzan. ${ }^{15}$ (ii) To simulate the quartz-crystal microbalance measurement, one may let the substrate atoms oscillate laterally and determine the induced lateral vibration of the adatoms. This method was also used by Cieplak et al. ${ }^{13,14}$ (iii) One can apply a constant force to the adatoms up to a specific point in time and then switch it off. If the friction is viscous, the decay of the steady-state velocity of the adsorbate is proportional to $e^{-\bar{\eta} t}$. (iv) Finally, one may determine $\bar{\eta}$ from the thermal equilibrium autocorrelation function of the center of mass velocity of the overlayer. The latter two methods were employed by Tomassone et al. ${ }^{16}$

In the present work, we also apply a constant lateral force $\mathbf{F}$ to the adatoms and determine the steady-state velocity that is reached after a sufficiently long time. The simulations are based on the Langevin equation

$$
m \ddot{\mathbf{r}_{i}}+m \eta \dot{\mathbf{r}_{i}}=-\frac{\partial U}{\partial \mathbf{r}_{i}}-\frac{\partial V}{\partial \mathbf{r}_{i}}+\mathbf{f}_{i}+\mathbf{F},
$$

where $\mathbf{f}_{i}$ is a stochastically fluctuating force describing the effect of the irregular thermal motion of the substrate on the $i$ th adatom. The components of $\mathbf{f}_{i}$ are related to the microscopic friction $\eta$ via the fluctuation-dissipation theorem

$$
\left\langle f_{i}^{\alpha}(t) f_{j}^{\beta}(0)\right\rangle=2 m k_{\mathrm{B}} T \eta_{\alpha \beta} \delta_{i j} \delta(t) .
$$

The microscopic friction tensor $\eta_{\alpha \beta}$ is assumed to be diagonal, with independent elements $\eta_{\|}$and $\eta_{\perp}$.

To solve Eq. (3.7) the time variable is discretized with a step duration $0.01 t_{0}$, where the natural time unit is $t_{0}$ $=r_{0}(m / \epsilon)^{1 / 2}=3.84 \mathrm{ps}$. The simulations are carried out using the integration procedure suggested by Tully et al. ${ }^{19}$ Typically, thermalization was reached after $10^{2}-10^{3} t_{0}$. The drift friction $\bar{\eta}$ was derived using the definition $\bar{\eta}$ $=F /(m\langle\mathrm{v}\rangle)$, where the external force $F$ was applied in the $x$ direction on each adsorbate, resulting in the (time averaged) drift velocity $\langle\mathrm{v}\rangle$. On $\operatorname{Ag}(100)$, the basic unit was taken as a square containing $12 \times 12$ or $24 \times 24$ substrate atoms. Similar unit cells were employed for $\operatorname{Ag}(111)$. These cells were then repeated assuming periodic boundary conditions.

Except for the form of the substrate potential, the model outlined above is very similar to the ones used in earlier simulations. ${ }^{13-16}$ The main difference between the treatments of Refs. 13-16 resides in the interpretation of the microscopic friction of a single Xe adatom. In the work by Cieplak et al. ${ }^{13,14}$ this friction plays the role of a thermostat that allows to establish a constant temperature $T$ within the overlayer. Since the direction of this single-site friction is chosen orthogonal to the direction of the external force, it was claimed not to affect the net sliding friction of the overlayer. Instead, in the work by Persson and Nitzan ${ }^{15}$ this thermostat was given a specific microscopic origin. In particular, $\eta_{\|}$ coincides with the lateral friction of individual Xe atoms due to excitation of electron-hole pairs in the substrate, i.e., $\eta_{\|}$ $=\eta_{\mathrm{el}}$. According to the theoretical estimates and the resistivity measurements discussed in Sec. II, this coefficient should be about $\eta_{\|} \approx 0.5 \mathrm{~ns}^{-1}$. Finally, in the work by Tomassone et al. ${ }^{16}$ the simulations were carried out in the absence of a thermostat or any other single-site friction forces.

In principle, electronic processes also contribute to the friction of the perpendicular motion of single adatoms. As pointed out in Sec. II, density functional calculations yield $\eta_{\perp} \approx 1.74 \mathrm{~ns}^{-1}$. However, this contribution is much smaller than the normal friction induced by phonon excitation, which was estimated by Persson and Nitzan ${ }^{15}$ to be roughly $\eta_{\perp}$ $=260 \mathrm{~ns}^{-1}$. Because of the present uncertainties in the evaluation of both $\eta_{\|}$and $\eta_{\perp}$, we have carried out simulations for a whole range of these parameters, in order to illustrate the sensitivity of the net sliding friction to the microscopic processes governing the behavior of individual Xe atoms.

\section{RESULTS AND DISCUSSION}

In this section we first discuss the sliding friction of a monolayer of $\mathrm{Xe}$ atoms on $\mathrm{Ag}$ and then address the coverage dependence. In the final part, we compare these results with the QCM data. The temperature of $T=77.4 \mathrm{~K}$, which we adopt, corresponds to the one used in the measurements by Krim et al. ${ }^{3}$ We define the coverage $\Theta=1$ as the fully compressed monolayer ${ }^{20}$ with density $n_{a}=0.0597 \AA^{-2}$. On $\operatorname{Ag}(100)$ this amounts to $N=72 \mathrm{Xe}$ atoms within the basic $12 a \times 12 a$ unit cell $(N=288$ within the $24 a \times 24 a$ cell $)$. The uncompressed monolayer has a slightly lower density, $n_{a}$ $=0.0565 \AA^{-2}$, i.e., $\Theta=0.94(N=68$ atoms within the $12 a$ $\times 12 a$ unit cell). The remaining parameters required in the simulations, which we report below, have been specified in the preceding sections.

\section{A. Corrugation dependence of the sliding friction for an uncompressed Xe layer}

In Fig. 2 we display typical results of the center of mass velocity, in the direction of the applied force, as a function of time. The examples are for $N=68$ on the (100) surface and the constant external force on each of the adsorbates $F$ $=0.001 \epsilon / r_{0}$ is applied after $100 t_{0}$ as indicated by the arrow. The single-atom friction coefficients are $\eta_{\|}=0$ and $1.3 \mathrm{~ns}^{-1}$, 

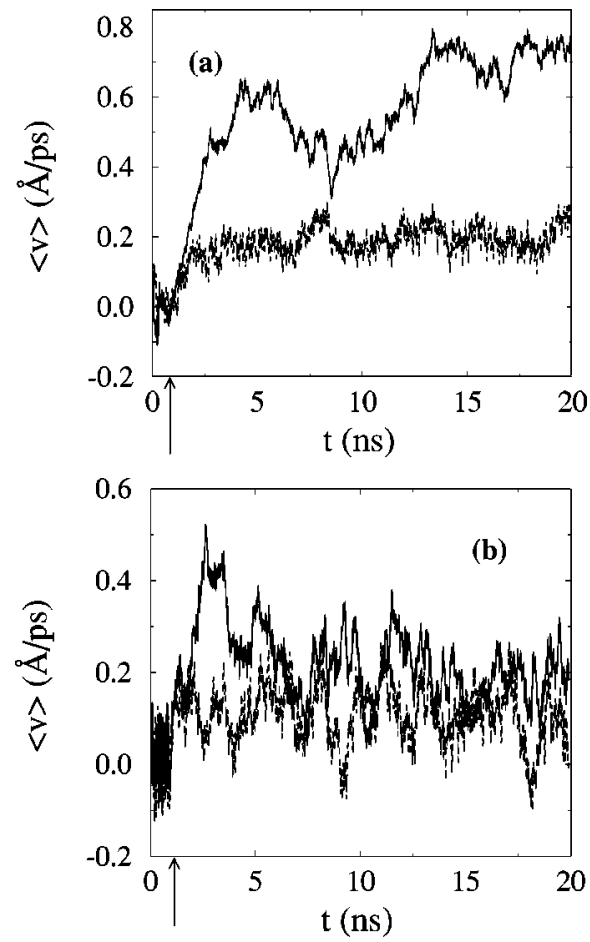

FIG. 2. Center of mass velocity (in units $\AA / p s$ ) of a Xe layer on $\operatorname{Ag}(100)$ as a function of time. The coverage corresponds to an uncompressed monolayer at $\Theta=0.94$ or $n_{a}=0.0565$ atoms $/ \AA^{2}$. The arrow indicates the time $\left(t=100 t_{0}=384 \mathrm{ps}\right)$ at which the external force $F=0.001 \epsilon / r_{0}=4.2 \times 10^{-3} \mathrm{meV} / \AA$ is applied. Upper curves are for $\eta_{\|}=0$, lower curves correspond to $\eta_{\|}=1.3 \mathrm{~ns}^{-1}$. (a) $u_{0}$ $=0.95 \mathrm{meV}$; (b) $u_{0}=1.9 \mathrm{meV}$.

with $\eta_{\perp}=260 \mathrm{~ns}^{-1}$. The corrugation amplitude is $u_{0}=0.95$ and $1.9 \mathrm{meV}$. One of the conclusions of the present work can be derived directly from this figure. In Fig. 2(a), for a relatively low corrugation amplitude $u_{0}=0.95 \mathrm{meV}$, the effect of the single-site friction coefficient $\eta_{\|}$is evident: suppressing it, the center of mass velocity increases and so do the fluctuations. But the effect of the suppression is attenuated when the corrugation amplitude is bigger, as is the case in Fig. 2(b) for $u_{0}=1.9 \mathrm{meV}$. Thus, the relative importance of phononic or electronic dissipation channels depends crucially on the magnitudes of $u_{0}$ and $\eta_{\|}$.

The validity of the linear response regime can be checked in Fig. 3, where we show the center of mass velocity $\langle v\rangle$, time averaged over $4000 t_{0}$ after the transient period following the application of the external force $\mathbf{F}$, as a function of $F$. The approximate linear relation $F=m \bar{\eta}\langle\mathrm{v}\rangle$ is apparent in all cases, indicating that the system indeed obeys a viscous force law. The full lines represent weighted linear regressions of the $\langle\mathrm{v}\rangle \sim F$ relation, which we use to derive the net sliding friction $\bar{\eta}$.

Figure 4 summarizes our results for $\bar{\eta}$ as a function of the corrugation amplitude $u_{0}$ and for different values of the lateral electronic single-atom friction $\eta_{\|}$. The overall sliding friction is approximately of the form

$$
\bar{\eta}=\eta_{\mathrm{el}}+c u_{0}^{2},
$$

with $\eta_{\mathrm{el}}=\eta_{\|}$. The intra-adsorbate phonon contribution $\eta_{\mathrm{ph}}$ $=c u_{0}^{2}$ varies quadratically with the corrugation amplitude $u_{0}$

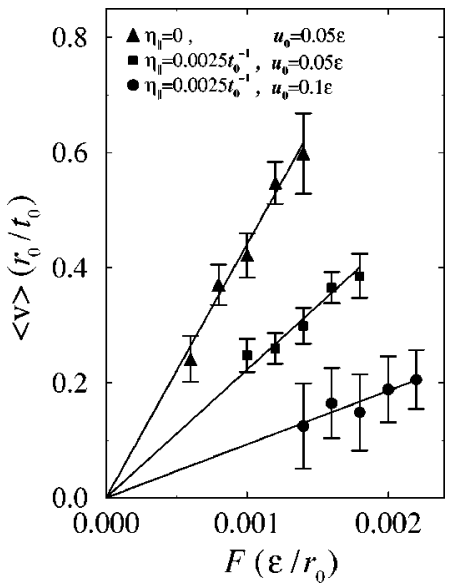

FIG. 3. Average center of mass velocity $\langle\mathrm{v}\rangle$ (in units of $r_{0} / t_{0}$ ) as a function of external force $F$ (in units of $\epsilon / r_{0}$ ) showing the linear viscous regime for different values of the input parameters $\eta_{\|}$ and $u_{0}$. Each point was obtained by time averaging over a long steady state run and over two independent runs. Error bars were estimated from the fluctuations of the center of mass velocity as can be seen in Fig. 2. Straight lines are linear fits of points accounting for error bars. The values of $\bar{\eta}$ are as follows (from top to bottom): $0.0023 / t_{0}, 0.0045 / t_{0}$, and $0.010 / t_{0}$.

and the coefficient $c$ depends weakly on the coefficient $\eta_{\|}$ and on the crystal structure of the substrate. This can be seen by comparing the results shown in Figs. 4(a) and 4(b) for the $\operatorname{Ag}(100)$ and $\operatorname{Ag}(111)$ surfaces, respectively. Although the overall variation of $\bar{\eta}$ is similar for both faces, the coefficient $c$ is slightly larger for $\operatorname{Ag}(111)$ than for $\operatorname{Ag}(100)$, indicating that $\bar{\eta}$ is governed by the total barrier height $\left[4.5 u_{0}\right.$ for (111) vs $u_{0}$ for (100)], rather than by the corrugation barrier between neighboring hollow sites [0.5 $u_{0}$ for (111) vs $2 u_{0}$ for (100); see previous section]. This trend is plausible since, at monolayer coverage, the Xe atoms are not free to move along paths between nearest potential minima but are forced across potential maxima. We note here that, in the $\eta_{\|}=0$ case, a quadratic variation of $\bar{\eta}$ with $u_{0}$ was also found by Cieplak et al. ${ }^{13,14}$

In order to demonstrate the weak dependence of the coefficient $c$ on $\eta_{\|}$, we collapse in Fig. 5 the data obtained for different $\eta_{\|}$into one curve by plotting

$$
\eta_{\mathrm{ph}}=\bar{\eta}-\eta_{\|}
$$

The full lines correspond to a quadratic fit of the data points. According to Figs. 5(a) and 5(b), we deduce for the (100) and (111) faces $c \approx 0.6 t_{0}^{-1} \epsilon^{-2} \approx 0.42 \mathrm{~ns}^{-1} \mathrm{meV}^{-2}$ and $c$ $\approx 0.8 t_{0}^{-1} \epsilon^{-2} \approx 0.56 \mathrm{~ns}^{-1} \mathrm{meV}^{-2}$, respectively.

The results shown in Figs. 4 and 5 correspond to $\eta_{\perp}$ $=1 / t_{0}=260 \mathrm{~ns}^{-1}$. Simulations for different values of $\eta_{\perp}$ in the range $26 \mathrm{~ns}^{-1} \leqslant \eta_{\perp} \leqslant 260 \mathrm{~ns}^{-1}$ yield nearly the same overall sliding friction, confirming the results of previous studies which suggested that the friction in the direction of the external force is essentially independent of the thermostat or the single-site friction in the orthogonal direction. ${ }^{13-15}$

The simulations discussed so far were carried out for 68 $\mathrm{Xe}$ atoms within substrate unit cells corresponding to $12 a$ 

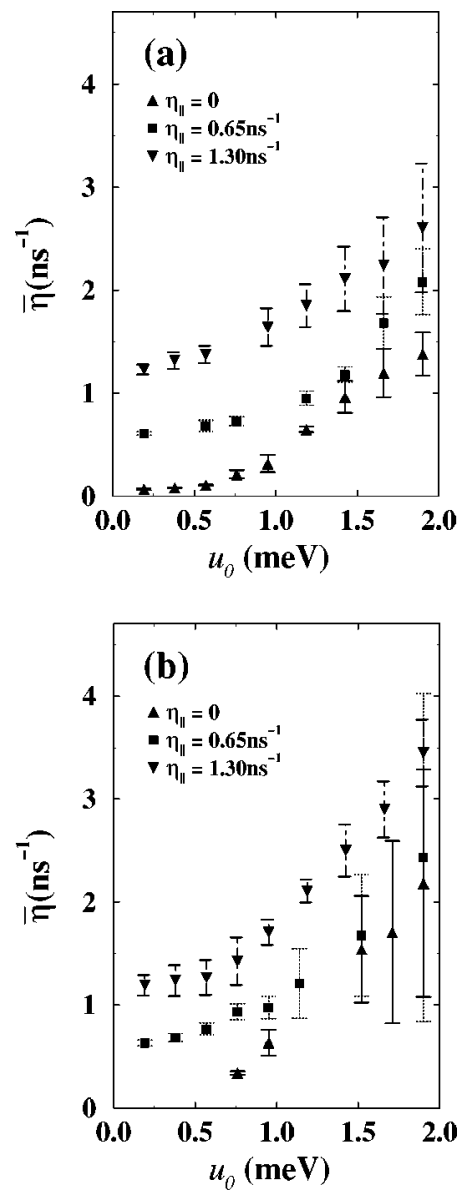

FIG. 4. Net sliding friction of Xe layer on $\mathrm{Ag}$ as a function of corrugation amplitude $u_{0}$. The coverage is $\Theta=0.94$. The results are shown for three values of the lateral single-atom electronic friction, indicated by the symbols: $\eta_{\|}=\eta_{\mathrm{el}}=0,0.0025 / t_{0}$, and $0.0050 / t_{0}$. $\eta_{\perp}=1.0 / t_{0}$ in all cases. (a) (100) surface; (b) (111) surface. The error bars were estimated on the basis of the linear weighted fits of Fig. 3, taking into consideration the center of mass velocity fluctuations of each individual run.

$\times 12 a$ for $\mathrm{Ag}(100)$ and $12 a \times 14 a \sqrt{3} / 2$ for $\mathrm{Ag}(111)$. Remarkably, we observed pronounced finite-size effects associated with the small dimension of these unit cells. This is illustrated for $\operatorname{Ag}(100)$ in Fig. 6, where $\eta_{\text {ph }}$ is plotted as a function of $u_{0}$. In general the Xe atoms form approximate hexagonal layers. Their orientation, however, tends to prefer two angles: with rows of Xe atoms nearly parallel to the $x$ axis, or nearly parallel to the $y$ axis. We denote these phases by $\alpha$ and $\beta$, respectively. Since these orientations persist even in the absence of the substrate corrugation $\left(u_{0}=0\right)$ and for vanishing external force, it is clear that they are a consequence of the limited size of the unit cell. The reason is that it is not possible to accomodate truly incommensurate hexagonal overlayers, at arbitrary angular orientations, in a rectangular unit cell that is periodically repeated.

In order to reduce these finite-size effects, we have performed simulations for much larger substrate cells [e.g., 288 $\mathrm{Xe}$ atoms in a $24 a \times 24 a$ cell on $\mathrm{Ag}(100)]$. Qualitatively, the net sliding friction for these systems agrees rather well with the earlier results, but the tendency for orientational alignment of the Xe layer is greatly reduced.
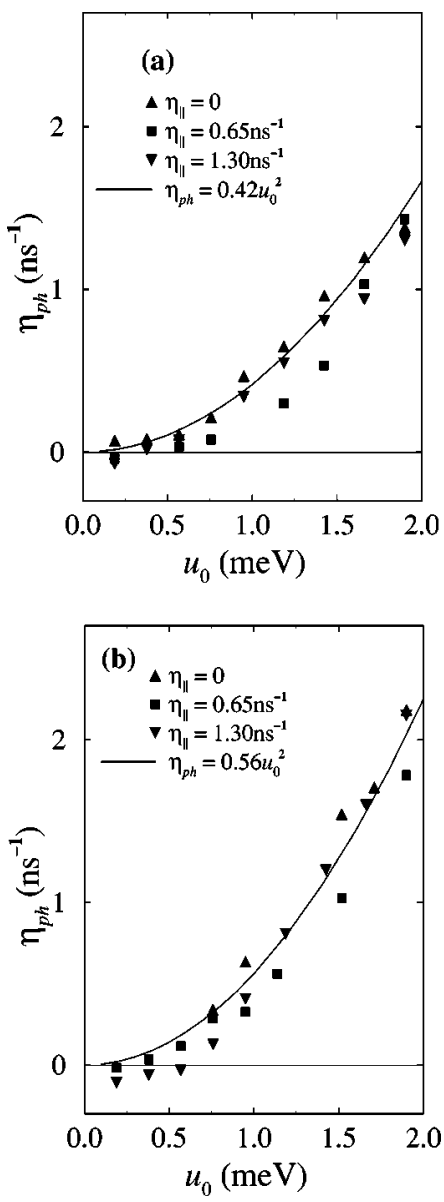

FIG. 5. Phonon contribution to the sliding friction of Xe layer on Ag obtained as $\bar{\eta}-\eta_{\|}$, as a function of corrugation amplitude $u_{0}$. Symbols as in Fig. 4. Full lines are quadratic fits accounting for errors of points. Error bars are the same as Fig. 4 but are suppressed here for clarity. (a) (100) surface; (b) (111) surface.

\section{B. Coverage dependence of sliding friction}

As mentioned above, the coefficient $c$ of the quadratic term in Eq. (4.1) depends strongly on overlayer coverage.

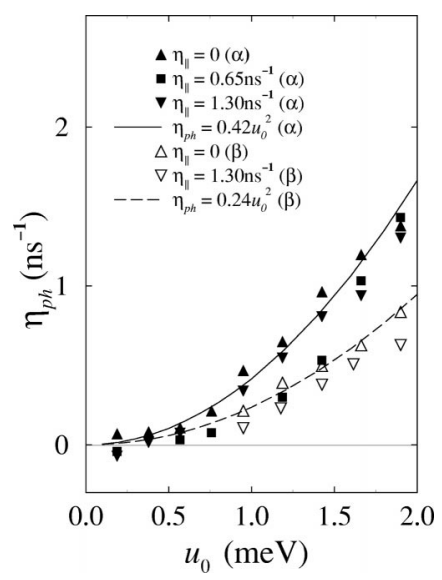

FIG. 6. Phonon contribution to the sliding friction of Xe layer on $\operatorname{Ag}(100)$ obtained as $\bar{\eta}-\eta_{\|}$, as a function of corrugation amplitude $u_{0}$. Symbols and quadratic fits as in Fig. 5. Full symbols and full line: $\alpha$ phase, open symbols and dashed line: $\beta$ phase, corresponding to different angular orientation of Xe monolayer. 

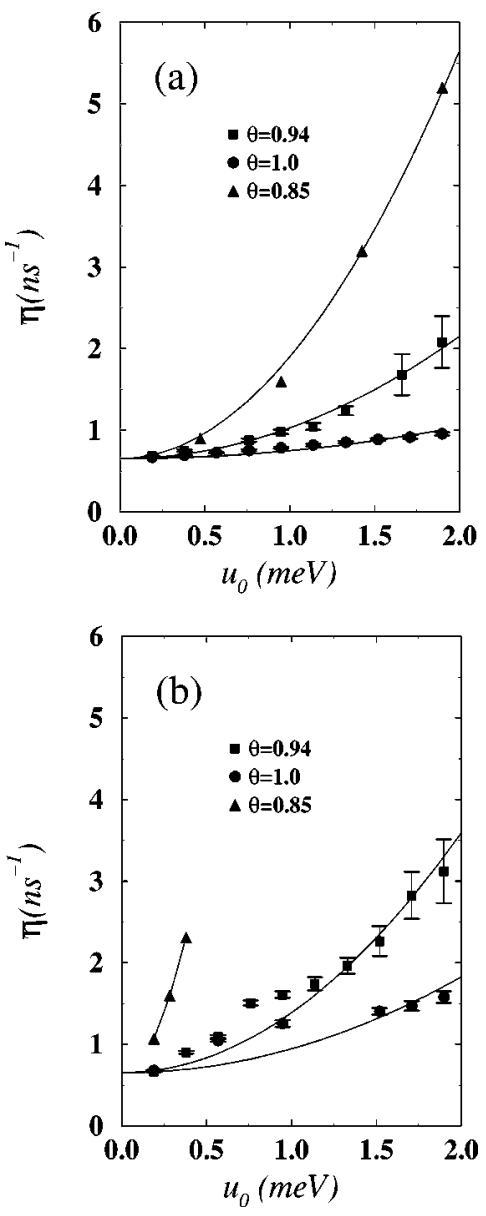

FIG. 7. Net sliding friction $\bar{\eta}$ of Xe layers on $\mathrm{Ag}$ as a function of corrugation amplitude $u_{0}$ for coverages $\Theta=0.85,0.94,1.0$. (a) (100) surface; (b) (111) surface. $\eta_{\|}=0.65 \mathrm{~ns}^{-1}$.

We illustrate this point in Fig. 7, where the net sliding friction of the uncompressed monolayer $(\Theta=0.94)$ is compared with that for a fully compressed Xe layer $(\Theta=1.0)$ and for a less dense layer $(\Theta=0.85)$. The compression is seen to lead to a greatly reduced phonon-induced contribution to $\bar{\eta}$. On $\operatorname{Ag}(100)$ as well as $\operatorname{Ag}(111)$, the magnitude of $c$ is reduced by more than a factor of 2 . A strong reduction of dissipation via phonons upon compression is to be expected since the $\mathrm{Xe}$ atoms are much less free to vibrate about the equilibrium positions. The opposite trend is found if the coverage is reduced below that of the uncompressed monolayer. For Xe on $\operatorname{Ag}(100)$ at coverage $\Theta=0.85$, the coefficient $c$ is about $1.25 \mathrm{~ns}^{-1} \mathrm{meV}^{-2}$, i.e., three times larger than for $\Theta=0.94$. An even more dramatic increase of $c$ is found on $\operatorname{Ag}(111)$. The strong enhancement of $\bar{\eta}$ is caused by the higher probability of exciting atomic vibrations in the less dense overlayer phase.

Concerning the overall variation of $\eta_{\mathrm{ph}}$ in the coverage range $\Theta=0.85 \cdots 1.0$, on $\operatorname{Ag}(100)$ for $u_{0}=0.95 \mathrm{meV}$ the variation is about $1 \mathrm{~ns}^{-1}$, while for $u_{0}=0.5 \mathrm{meV}$ this variation diminishes to less than $0.3 \mathrm{~ns}^{-1}$, and for $u_{0}=1.4 \mathrm{meV}$ it is larger than $2 \mathrm{~ns}^{-1}$. On $\operatorname{Ag}(111)$, in the same coverage range, $u_{0}=0.3 \mathrm{meV}$ yields a variation of $\eta_{\mathrm{ph}}$ of about $1 \mathrm{~ns}^{-1}$, whereas $u_{0}=0.2 \mathrm{meV}\left(u_{0}=0.4 \mathrm{meV}\right)$ lead to much smaller (larger) variations. Therefore $\eta_{\mathrm{ph}}$ is sensitive to the substrate topology.
In view of the great sensitivity of $\eta_{\mathrm{ph}}(\Theta)$ with respect to the corrugation amplitude, it would be of interest to have more accurate independent experimental and/or theoretical determinations of the substrate-adsorbate potential. In principle, the corrugation amplitude may be estimated from the lateral vibrational frequency $\omega_{\|}$of adsorbed Xe, which can be detected using inelastic $\mathrm{He}$ scattering. For $\mathrm{Xe}$ on $\mathrm{Cu}(111)$, one finds $\omega_{\|} \approx 3 \pm 1 \mathrm{~cm}^{-1} \cdot{ }^{21}$ Using the relation $u_{0}$ $=m \omega_{\|}^{2} / k^{2}$, one obtains values of $u_{0}$ in the approximate range between 0.5 and $2 \mathrm{meV}$. From Eq. (4.1) it follows that $\eta_{\mathrm{ph}}$ varies with the fourth power of $\omega_{\|}$. This is born out by our simulations which show that such barriers yield a phononinduced friction in a very wide range, $\eta_{\mathrm{ph}} \approx 0.1 \cdots 5.0 \mathrm{~ns}^{-1}$, if the coverage is varied between 1.0 and 0.85 .

\section{Comparison with experiment}

As pointed out in Sec. I, the quartz-crystal microbalance measurements by Krim et l. $^{3}$ for thin Xe films on $\mathrm{Ag}(111)$ show that the slip time $\tau=1 / \bar{\eta}$ exhibits a characteristic dependence on Xe coverage (see Fig. 1). Close to one monolayer, the slip time reaches a minimum of about $\tau \approx 0.8 \mathrm{~ns}$ and then increases to about $2.0 \mathrm{~ns}$ within a coverage range of less than 0.3 monolayers. In this section we focus on this coverage range (roughly the "compression region") since it provides the most challenging aspect of the data. The interpretation of the slip time at submonolayer coverages in terms of simulations based on small repeated cells is less reliable because they do not account for the formation of islands. Also, the presence of defects and steps which are not included in the theory then plays a larger role. On the other hand, beyond full monolayer coverage, the observed slip time shows a much weaker coverage dependence related to additional phonon dissipation in the partly filled second monolayer.

In Ref. 16 it is stated that the minimum of $\tau$ corresponds to the uncompressed monolayer at $n_{a} \approx 0.0563 \AA^{-2}$ and that the subsequent steep rise reflects the suppression of intraadsorbate phonon excitations as the Xe layer becomes more compressed (as noted above, the compressed phase is only $6 \%$ denser than the uncompressed layer ${ }^{20}$ ). This coverage assignment implies that the slip time increases (i) when the coverage is reduced below that of the uncompressed monolayer and (ii) when the coverage is increased beyond that of the compressed layer. However, both of these implications are implausible since in general incommensurate solid layers slide more easily than fluids. ${ }^{13-15}$ Thus, the slip time should decrease rather than increase (i) when the coverage is lowered below that of the uncompressed layer and (ii) when it is increased beyond that of the compressed layer, i.e., when the second layer is beginning to adsorb. Since absolute coverage calibration in the experiment is difficult, there exists a clearly appreciable uncertainty concerning the measured sliding friction of a monolayer. In fact, it would appear more plausible to associate the minimum of the slip time not with the uncompressed layer but with a slightly lower coverage. Part of the observed steep rise of $\tau$ would then correspond to the reduction of intraoverlayer phonon processes upon reaching the uncompressed layer coverage, and the remaining rise should reflect the additional phonon reduction due to com- 

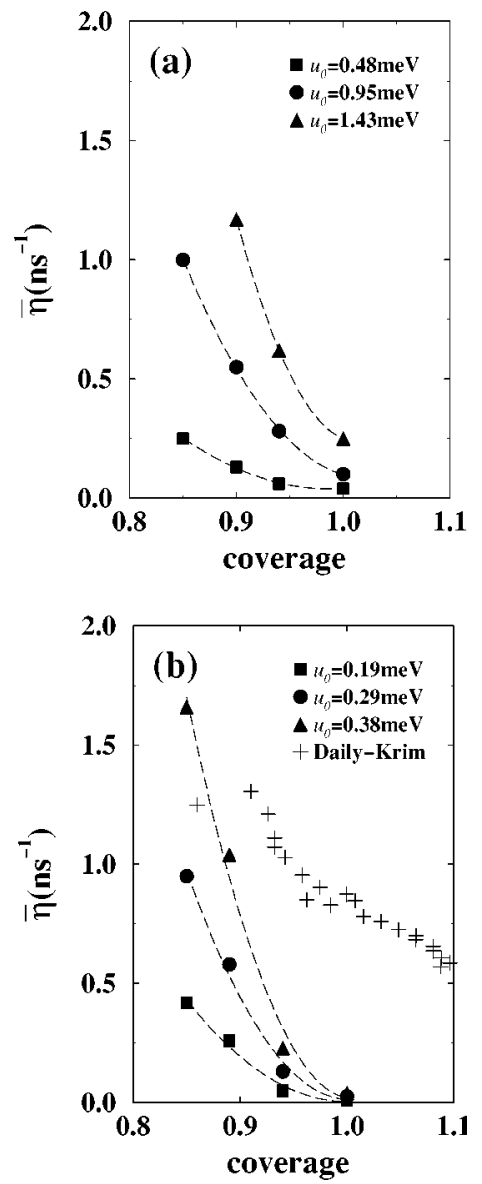

FIG. 8. Calculated phonon friction $\eta_{\mathrm{ph}}(\Theta)$ of $\mathrm{Xe}$ on $\mathrm{Ag}$ as a function of coverage $\Theta$ for several corrugation amplitudes $u_{0}$. (a) (100) surface; (b) (111) surface. The dashed lines are guides to the eye. Panel (b) also shows the measured sliding friction $\bar{\eta}=1 / \tau$ in the compression region where the slip time $\tau$ exhibits the characteristic positive slope (see Fig. 1).

pression. The maximum of the observed slip time should approximately correspond to the fully compressed phase. According to these arguments, the uncompressed monolayer might have $\tau \approx 1.3 \cdots 1.6 \mathrm{~ns}$, giving $\bar{\eta} \approx 0.6 \cdots 0.8 \mathrm{~ns}^{-1}$.

In Fig. 8 the phonon friction $\eta_{\mathrm{ph}}(\Theta)$ is plotted as a function of coverage, in the range $\Theta=0.85 \cdots 1.0$, for several values of the corrugation amplitude $u_{0}$. For ease of comparison with the experimental results, we include in Fig. 8(b) the measured $\bar{\eta}=1 / \tau$ as a function of coverage in the region near one monolayer where $\tau$ exhibits the characteristic positive slope. To account for the uncertainty of the measured coverage, and for the expected behavior of the sliding friction as discussed above, a shift of the data to lower coverages, by about $0.1 \cdots 0.15$ monolayer, should be allowed for. The coverage dependence of $\bar{\eta}$ in the range shown can be characterized by two important features: the overall magnitude and the slope. The comparison of $\bar{\eta}$ with the calculated results shows that none of the functions $\eta_{\mathrm{ph}}(\Theta)$ obtained for different corrugation amplitudes $u_{0}$ provides an adequate description of the data. However, for $u_{0}=0.19 \mathrm{meV}$, the calculated $\eta_{\mathrm{ph}}$ has roughly the right slope even though its magnitude at full monolayer coverage is far too small. If $u_{0}$ is increased to $0.3 \cdots 0.4 \mathrm{meV}$ the slope of $\eta_{\mathrm{ph}}(\Theta)$ becomes rapidly larger than that of the measured $\bar{\eta}$ while the magnitude near monolayer coverage is still too small. Only when $u_{0}$ is increased to much larger values does $\eta_{\mathrm{ph}}$, in the compressed phase, match the observed $\bar{\eta}$, but the slope of $\eta_{\mathrm{ph}}(\Theta)$ then is much larger than observed.

As shown in Fig. 8(a), a similar picture is found on $\mathrm{Ag}(100)$. Although the detailed dependence of $\eta_{\mathrm{ph}}(\Theta)$ on the corrugation differs appreciably from the one obtained for the (111) face, near monolayer coverage, $\eta_{\mathrm{ph}}$ remains less than $0.1 \mathrm{~ns}^{-1}$ as long as $u_{0}<1 \mathrm{meV}$, with a moderate slope for $\Theta<1$. Only if $u_{0}$ is increased to $2 \mathrm{meV}$ we find $\eta_{\mathrm{ph}}$ $\approx 0.5 \mathrm{~ns}^{-1}$ in the compressed phase. The slope of $\eta_{\mathrm{ph}}(\Theta)$, however, then quickly becomes extremely large.

Thus, our results imply that it is not possible to simultaneously describe the magnitude and slope of the observed variation of $\bar{\eta}(\Theta)$ exclusively in terms of phonon dissipation. Only by adding an electronic friction contribution (which in the narrow range shown in Fig. 8 can be assumed to be independent of coverage) does the sum $\eta_{\mathrm{el}}+\eta_{\mathrm{ph}}(\Theta)$ reproduce the measured variation of $\bar{\eta}(\Theta)$. The comparison with the data suggests that $\eta_{\mathrm{el}} \approx 0.5 \mathrm{~ns}^{-1}$ together with $u_{0}$ $\approx 0.2 \mathrm{meV}$ (total barrier height $\approx 0.9 \mathrm{meV}$ ) provides a qualitative description of the sliding friction measured on $\operatorname{Ag}(111)$ - if one allows a shift of the data of 0.14 monolayer to lower coverages, of course. As discussed in Sec. II, an overall single-atom electronic friction coefficient of this magnitude is in agreement with surface resistivity measurements and independent theoretical estimates.

Interestingly, our findings for $\mathrm{Xe} / \mathrm{Ag}$ differ from those on $\mathrm{Kr} / \mathrm{Au}$. As recently shown by Robbins and $\mathrm{Krim}^{22}$ the $\mathrm{Kr} / \mathrm{Au} \mathrm{QCM}$ data up to monolayer coverage can be fitted rather well assuming negligible electronic friction. A finite electronic friction would require a correspondingly smaller substrate corrugation $u_{0}$ which, in turn, would yield too low values of the phonon friction at submonolayer coverages. We note, however, that the analysis of the low-coverage data is uncertain since the simulations are limited to repeated finite cells and do not include the effect of defects or steps, nor do they properly describe the formation of islands which most likely occur under experimental conditions. Thus, if one focuses on the slip time in the less problematic region near monolayer coverage, i.e., roughly $0.85 \leqslant \Theta \leqslant 1$, the analysis in Ref. 22 indicates that the inclusion of a finite electronic friction and a slightly reduced corrugation $u_{0}$ is not incompatible with the data.

In addition, we point out that electronic friction of $\mathrm{Kr}$ on $\mathrm{Au}$ indeed ought to be weaker than for $\mathrm{Xe}$ on $\mathrm{Ag}$ for the following reasons. Since the $\mathrm{Au} d$ bands lie only $2 \mathrm{eV}$ below the Fermi level, in contrast to $4 \mathrm{eV}$ for $\mathrm{Ag}$, the larger $s-d$ hybridization diminishes the polarizability of the $s$ electron tails decaying into the vacuum region and thereby reduces the probability of exciting substrate electron-hole pairs via adsorbed particles. The larger work function of $\mathrm{Au}$ (5.3 compared to $4.8 \mathrm{eV}$ ) also contributes to a stiffer, less polarizable electronic density tail. ${ }^{11,12}$ Moreover, since the atomic polarizability of $\mathrm{Kr}$ is about $40 \%$ smaller than that of $\mathrm{Xe},{ }^{23}$ the leading van der Waals part of the friction coefficient should be much smaller than for Xe on Ag. For the same physical reasons, the contributions associated with the Pauli repulsion and the covalency of the adatom bond should also be 
smaller. The overall electronic coupling of $\mathrm{Kr}$ on $\mathrm{Au}$ should therefore be significantly weaker than for $\mathrm{Xe}$ on $\mathrm{Ag}$. Thus, great caution is required when comparing electronic excitation processes on different adsorption systems - even in the case of rare gas atoms on noble metal surfaces.

The comparison of Figs. 8(a) and 8(b) suggests that, for the same corrugation amplitude $u_{0}, \operatorname{Ag}(111)$ yields a larger phonon friction than $\operatorname{Ag}(100)$. This trend seems at first surprising since the close-packed (111) face is generally regarded as the smoothest of the low-index faces of an fcc crystal. On the other hand, as pointed out in Sec. III, the potential barrier between neighboring hollow sites is $2 u_{0}$ for the (100) face and $0.5 u_{0}$ for the (111) face, while the total barrier between hollow sites across the substrate lattice sites is $4 u_{0}$ for the (100) face and $4.5 u_{0}$ for (111). Thus, the (111) surface has sharper potential maxima than the (100) face. Since atoms of an incommensurate overlayer sample not only the potential minima but the entire substrate surface, it is indeed plausible that $\mathrm{Ag}(111)$ causes more Xe intralayer phonon friction than $\operatorname{Ag}(100)$. According to this, the topology of the substrate is a relevant feature if one want to make a comparison with the experiments.

In their analysis of an uncompressed Xe layer on $\mathrm{Ag}(100)$, Persson and Nitzan ${ }^{15}$ used $u_{0}=0.95 \mathrm{meV}$ and obtained $\eta_{\mathrm{ph}} \approx 0.01 \mathrm{~ns}^{-1}$ (for $\eta_{\|}=0.62 \mathrm{~ns}^{-1}$, they find $\eta_{\|} / \bar{\eta}$ $=0.98 \pm 0.04$ ). From this result, they concluded that the net friction at monolayer coverage is mainly of electronic origin. Instead, for the same parameters, we find $\eta_{\mathrm{ph}} \approx 0.3 \mathrm{~ns}^{-1}$, suggesting a phonon friction roughly half as large as the electronic contribution.

On the other hand, Tomassone et al. ${ }^{16}$ assumed a total barrier height of $2 \mathrm{meV}$ on $\mathrm{Ag}(111)$, implying in our notation $u_{0}=0.45 \mathrm{meV}$. On the basis of the simulations it was concluded that phonon friction dominates over possible electronic contributions. For an uncompressed Xe layer, these authors obtained a phonon friction $\eta_{\mathrm{ph}} \approx 1.8 \mathrm{~ns}^{-1}$, which is considerably larger than what we find for the same parameters. According to Fig. 2 of Ref. 16, in the compression region, the calculated slip time for $u_{0}=0.45 \mathrm{meV}$ increases extremely rapidly in agreement with our results. This increase, however, is much steeper than what is seen in the experiment. Thus, a weaker corrugation, combined with a finite electronic friction might, in fact, provide a better fit of the data in this coverage range. The analysis of the submonolayer data is again more complicated for the reasons discussed earlier.

\section{CONCLUSION}

We have performed molecular dynamics simulations for Xe layers on $\operatorname{Ag}(100)$ and $\operatorname{Ag}(111)$ in order to determine the net sliding friction in the presence of a constant lateral external force. We find that $\bar{\eta}$ depends sensitively on the corrugation amplitude $u_{0}$ of the $\mathrm{Xe} / \mathrm{Ag}$ interaction potential and on the single-adatom microscopic friction parameter $\eta_{\|}$. For coverages approaching one monolayer, the phonon-induced contribution $\eta_{\mathrm{ph}}(\Theta)$ decreases rapidly, in agreement with the strong positive slope of the observed slip time $\tau$. A detailed study of $\eta_{\mathrm{ph}}(\Theta)$ as a function of corrugation amplitude $u_{0}$ suggests that, close to monolayer coverage, it is not possible to consistently describe both the magnitude and slope of the measured $\bar{\eta}=1 / \tau$ without including a constant lateral electronic friction $\eta_{\mathrm{el}}$. Our simulations indicate that this electronic contribution should be about $0.5 \mathrm{~ns}^{-1}$ which is in qualitative agreement with surface resistivity measurements and independent theoretical estimates.

The overall picture that seems to be consistent with the available information is that the rapidly varying part of the observed sliding friction is due to excitation of atomic vibrations within the overlayer and that single-site electronic friction processes contribute a roughly coverage-independent background. For a fully compressed Xe layer, phonon dissipation is weaker than the electronic friction. As the coverage is reduced, however, phonon processes rapidly become important and eventually, near the minimum of the observed slip time, dominate over the electronic dissipation channel.

In summary, even though both experimental and theoretical results on sliding friction of $\mathrm{Xe}$ on $\mathrm{Ag}$ have inherent uncertainties, our analysis indicates that both mechanismsphononic and electronic dissipation-do contribute. The relative weight of these channels depends strongly on coverage in the crucial region near a full monolayer.

\section{ACKNOWLEDGMENTS}

One of the authors (A.L.) gratefully acknowledges the hospitality of the Facultad de Física, Universidad Católica, Chile, where this work was partially carried out, as well as financial support by the European Community. A.L. also thanks Professor Abe Nitzan for making available his molecular dynamics code and Dr. P. Ballone for several useful suggestions concerning the simulations. M.K. and S.G. were supported by the Fondo Nacional de Investigaciones Científicas y Tecnológicas (FONDECYT, Chile) under Grants No. 1971212 and No. 3950028, respectively, and by Fundación Andes.
*Electronic address: a.liebsch@fz-juelich.de

†Electronic address: sgonc@if.ufrgs.br

Electronic address: mkiwi@puc.cl

${ }^{1}$ F. P. Bowden and D. Tabor, Friction and Lubrication (Methuen, London, 1967).

${ }^{2}$ B. N. J. Persson, The Physics of Sliding Friction (Springer, Heidelberg, 1997).

${ }^{3}$ E. T. Watts, J. Krim, and A. Widom, Phys. Rev. B 41, 3466 (1990); A. Widom and J. Krim, Phys. Rev. E 49, 4154 (1994); J. Krim, D. H. Solina, and R. Chiarello, Phys. Rev. Lett. 66, 181 (1991).

${ }^{4}$ C. Daly and J. Krim, Phys. Rev. Lett. 76, 803 (1996); A. Dayo, W. Alnasrallah, and J. Krim, Phys. Rev. Lett. 80, 1690 (1998).
${ }^{5}$ C. Holzapfel, F. Stubenrauch, D. Schumacher, and A. Otto, Thin Solid Films 188, 7 (1990).

${ }^{6}$ W. L. Schaich and J. Harris, J. Phys. C 11, 65 (1981).

${ }^{7}$ F. Sols and F. Flores, Solid State Commun. 42, 687 (1982); F. Sols, F. Flores, and N. García, Surf. Sci. 137, 167 (1984).

${ }^{8}$ B. N. J. Persson, Phys. Rev. B 44, 3277 (1991).

${ }^{9}$ B. N. J. Persson and A. J. Volokitin, J. Chem. Phys. 103, 8679 (1995).

${ }^{10}$ J. B. Sokoloff, Phys. Rev. B 52, 5318 (1995).

${ }^{11}$ A. Liebsch, Phys. Rev. B 55, 13263 (1997).

${ }^{12}$ A. Liebsch, Electronic Excitations at Metal Surfaces (Plenum, New York, 1997). 
${ }^{13}$ M. Cieplak, E. D. Smith, and M. O. Robbins, Science 265, 1209 (1994).

${ }^{14}$ M. Cieplak, E. D. Smith, and M. O. Robbins, Phys. Rev. B 54, 8252 (1996).

${ }^{15}$ B. N. J. Persson and A. Nitzan, Surf. Sci. 367, 261 (1996).

${ }^{16}$ M. S. Tomassone, J. B. Sokoloff, A. Widom, and J. Krim, Phys. Rev. Lett. 79, 4798 (1997).

${ }^{17}$ G. C. Aers and J. E. Inglesfield, Surf. Sci. 217, 367 (1989).

${ }^{18}$ W. Steele, Surf. Sci. 36, 317 (1973).
${ }^{19}$ J. C. Tully, G. H. Gilmer, and M. Shugard, J. Chem. Phys. 71, 1630 (1979).

${ }^{20}$ P. Dai, T. Angot, S. N. Ehrlich, S. K. Wang, and H. Taub, Phys. Rev. Lett. 72, 685 (1994).

${ }^{21}$ J. Braun, D. Fuhrmann, A. Siber, B. Gumhalter, and Ch. Wöll, Phys. Rev. Lett. 80, 125 (1998).

${ }^{22}$ M. O. Robbins and J. Krim, Mater. Res. Bull. 23, 23 (1998).

${ }^{23}$ A. Zangwill and P. Soven, Phys. Rev. A 21, 1561 (1980). 